Revue d'histoire de l'Amérique française

REVUE D.HISTOIRE DE L'AMÉRIQUE FRANÇAISE

\title{
Plaintes contre l'administration de la justice (1807) (suite et fin)
}

Jean-Pierre Wallot

Volume 20, numéro 3, décembre 1966

URI : https://id.erudit.org/iderudit/302586ar

DOI : https://doi.org/10.7202/302586ar

Aller au sommaire du numéro

Éditeur(s)

Institut d'histoire de l'Amérique française

ISSN

0035-2357 (imprimé)

1492-1383 (numérique)

Découvrir la revue

Citer cet article

Wallot, J.-P. (1966). Plaintes contre l'administration de la justice (1807) (suite et fin). Revue d'histoire de l'Amérique française, 20(3), 366-379.

https://doi.org/10.7202/302586ar d'utilisation que vous pouvez consulter en ligne.

https://apropos.erudit.org/fr/usagers/politique-dutilisation/ 


\section{PLAINTES CONTRE L'ADMINISTRATION DE LA JUSTICE (1807) (fin)}

Le lendemain, 12 juin, cette fois en l'absence du juge de Bonne, un débat savoureux crépita à nouveau: devait-on lire la copie délivrée par le notaire ou celle du greffier ? Les juges anglais pataugeaient dans l'inconnu. Tous argumentaient en même temps, depuis Planté, J.-F. Perrault et les avocats jusqu'aux juges. $\AA$ ' bout, le juge en chef grogna finalement "que tout ce qu'on pouvait demander, c'était que le testament fut conservé, que soit qu'il fût publié de manière ou d'autre, cela était indifférent, qu'il ne fallait pas s'arrêter à toutes ces petites formes": aveu éloquent d'ignorance.

Ces arguties inutiles ne pouvaient qu'exaspérer avocats et plaideurs. Incompétents, les juges tâtonnaient, improvisaient leurs propres lois et règles au fur et à mesure des causes, selon leur inclination personnelle ou leur degré de lassitude. Pourtant les Britanniques imputaient à "l'obscurité" des lois françaises la confusion que secrétait en fait pareille administration de la justice. Les Canadiens s'indignaient: les impulsions du moment, les intérêts, les amitiés et l'orgueil des juges se substituaient au règne de la loi et plus précisément des lois françaises, méconnues et sujettes aux croisements les plus bizarres avec la Common Law.

Le 13 juin, la cour rejeta la demande de Me Planté à l'effet de publier aussi son "expédition" du testament de Duchesnay. C'était conjuguer l'insulte à l'illégalité — ou ce qui apparaissait tel aux avocats canadiens. C'était aussi une victoire de prestige

1 Voir notre Revue, XIX: 551-560; XX: 28-43, 281-290. Errata: à la page 282 de notre dernier no, il fallait lire, à la 21e ligne: "fallait-il ignorer toutes les autres sections conformes à la forme française et incompatibles avec l'anglaise ?". 
pour le greffier Perrault ${ }^{2}$ contre ses ennemis du Canadien. ${ }^{3}$ Un "Admirateur du Mécanicien" 4 ironisa à l'effet qu'il serait "bien plus convaincu de leur [aux "machines à justice"] utilité ou ... absurdité, si ... après leur avoir exposé les questions de droit... touchant le dépôt du testament de Mr. Duchesnay, elles rendaient un jugement non-conforme ou conforme au jugement déjà rendu". 5

Vers le même temps, Perrault l'aîné burinait un tableau pessimiste de l'administration de la justice, depuis la Conquête:

Depuis près de 30 ans que j'observe les décisions des Juges Britanniques sur ces vaines affaires de cette nature ou qui d'une manière ou d'autre tiennent au gouvernement de l'Eglise, ${ }^{6}$ de l'Etat \& prérogatives, j'ai toujours vu que la forme \& même nos lois étaient sacrifiées à la Politique constante des officiers de la Couronne et de là $j$ 'ai tout lieu de penser que les sindics ne réussiront pas plus en Appel qu'en cour inférieure. Néanmoins, et pour d'autres vues, il faut essayer...

Les machines du Canadien sont ingénieuses; mais ce comte du Testament m'ennuie; cependant dans les procédés de la Cour à ce sujet on voit encore l'esprit des Juges Anglais opposé à l'esprit \& à la lettre de nos lois et usages. Leur principe est de s'opposer: ils y réussissent presque toujours malgré les juges canadiens, tant dans les cours supérieures qu'inférieures...7

Perrault semble amer, presque découragé. Ce témoignage s'avère d'autant plus significatif que son auteur, un seigneur, un homme

2 Le greffier Joseph-François Perrault (1753-1844) était l'un des fondateurs du Courrier de Québec et, ne fût-ce qu'à cause de son poste, se ralliait au parti gouvernemental.

${ }^{3}$ Le Canadien, 11 juillet 1807 . Voir appendice $\mathrm{I}$.

4 Pour les articles du "Mécanicien", voir notre Revue, XIX: 551-560.

5 Le Canadien, 25 juillet 1807.

6 Perrault fait ici allusion à un procès survenu à la suite d'un conflit dans la paroisse Saint-André.

7 Perrault l'aîné à Olivier Perrault, Rivière Ouëlle, 14 juillet 1807, AC, collection Baby [M.G. 22/6i], 5-1: 187. L'allusion aux juges canadiens détonne un peu, étant donné leur attitude en général. 
à la fois indépendant d'esprit et très modéré, n'appartenait même pas au parti canadien. Par contre, il ne désespérait pas de l'avenir. A propos des nominations futures de juges, il avait écrit peu auparavant: "Il viendra un temps où les avocats canadiens distingués par leurs talents \& leurs mérites auront aussi leur tour. L'influence canadienne augmentera en raison des progrès que doit faire notre éducation et nos lumières." 8

Le 1er août, Le Canadien commenta - avec satisfaction cette fois - un récent jugement de la Cour d'appel. Celle-ci venait en effet de renverser une décision de la Cour du banc du roi de Montréal au sujet du temps "pour les oppositions à conserver" dans le cas des ventes par le shérif: la Cour de Montréal avait édicté un nouveau délai. Des arguments de la Cour d'appel, Le Canadien déduisait deux grandes conséquences, qu'il faut interpréter dans le contexte de l'affaire du testament de Duchesnay: " $1{ }^{\circ}$ Que les anciennes lois qui règlent les formes de procéder qui n'ont pas été expressément abrogées sont en force. $2^{\circ}$ Que toutes les Règles des Cours de justice de cette Province qui ne sont pas faites d'une manière aussi analogue que possible aux principes de celles de ces anciennes lois dont la lettre n'est pas en force, sont nulles de plein droit." 9

Les Canadiens ne se contentaient pas de geindre. Ils suggéraient également des remèdes aux maux qu'ils dénonçaient. Selon un "Amateur de la justice", dans aucun pays n'avait-on de lois meilleures ni de pire manière de les administrer. Tantôt on décidait selon l'équité, tantôt selon les lois françaises, tantôt selon les anglaises, souvent selon les deux systèmes entremêlés. Des causes semblables occasionnaient des jugements contradictoires. Dans les districts de Québec et de Montréal, pourquoi la Cour d'appel ne comprendrait-elle pas les conseillers et cinq juges, dont le juge en chef et quatre des "meilleurs jurisconsultes du pays". Six fois par année, cette cour reviserait tous les jugements des Cours du banc du roi. Il faudrait également

${ }^{8}$ Perrault l'aîné au même, 26 mai 1807, ibid., 119. Perrault s'illusionne quelque peu.

${ }^{9}$ Le Canadien, 1er août 1807. Voir appendice II. 
secourir les pauvres dans les cours inférieures. Au civil, la Cour du banc du roi serait composée de trois membres, dont le juge en chef du district; au criminel, du même nombre ainsi que du juge en chef de la province chaque fois que possible. Surtout, le Parlement devait retirer aux différentes cours "tous [leurs] pouvoirs législatifis \& par là empêcher nombre d'abus", dont celui d'abroger "des lois positives" - v.g. le pouvoir des "cours de quartiers de sessions" de décréter des "règlements de police" pouvant contredire des lois du Parlement. Dans les cours inférieures, un seul juge entendrait les causes mettant en jeu des sommes ne dépassant pas $£ 10$ st., puis jugerait toujours selon la loi et non selon l'équité: "sous ce prétexte les Cours peuvent donner des jugements... contraires à la loi et au bon sens". Trois juges de paix permanents tiendraient les "Cours de quartiers de sessions". Quant aux sessions de semaine, il faudrait y employer convenablement deux juges de paix à temps complet. Autrement, la cour se réunirait sporadiquement. Sans salaire, les juges vaquaient d'abord à leurs affaires, d'où les délais et les frais accrus. Enfin, une requête votée par les deux tiers de l'Assemblée devraĭt forcer le gouvernenr à destituer un mauvais juge. ${ }^{10}$

"Un souscripteur" releva ce dernier point. La constitution n'en soufflait mot. De plus, ici, les juges détenaient leur commission "durant plaisir" du roi et non, comme en Angleterre, "durant bonne conduite". Cependant, sans y être obligé, le gouverneur devrait déférer à une demande du Parlement et déplacer un juge accusé "après une investigation légale" de sa conduite. ${ }^{11}$

${ }^{10}$ Le Canadien, 21 novembre et 12 décembre 1807. Voir appendice III.

11 Le Canadien, 26 décembre 1807. Voir appendice IV. Le Canadien reviendrait plusieurs fois sur ce problème, surtout au début de 1808, en fustigeant habilement les juges. Ainsi, il feindrait d'encenser les vertus des juges en place. Mais dans l'avenir, il pourrait y en avoir des paresseux, incompétents, ambitieux, riches, etc. On pourrait certes chercher à les neutraliser par "l'impeachment"; mais les juges membres de l'Assemblée, "sacrifieront tour à tour les intérêts de la Couronne à ceux des sujets et les intérêts des sujets à ceux de la Couronne, selon qu'il leur conviendra". Les procès traîneraient en longueur ou seraient tranchés "à la hâte par un Greffier" - allusion à J.-F. Perrault. Le seul moyen 
"Un Ami du Bien" contestait la légende de "l'esprit de chicane des habitants". Dans le district de Montréal, par exemple, une seule cour présidait à toutes les causes ne dépassant pas $£ 10$ st. parmi une population de 100,000 habitants disséminés sur des centaines de milles. Encore ne siégeait-elle que cinq fois par année. D'où l'engorgement des causes et les délais ruineux qui rendaient les poursuites presque inutiles. L'auteur égrenait nombre d'exemples illustrant l'absurdité du système: d'autant plus que les débiteurs pouvaient recourir à des mesures dilatoires ajournant encore le jugement et son exécution. Une Cour hebdomadaire éliminerait la plupart de ces inconvénients. Dans les circonstances existantes, "la multitude des causes qui s'accumulent... met le Juge... hors d'état de donner à ses décisions toute l'attention nécessaire, les Avocats de rendre justice à leurs clients... et fournit en outre des moyens faciles à tous les plaideurs de mauvaise foi ou Chicaneurs d'éluder la Justice". ${ }^{12}$

L'année achevait. En prévision de la session prochaine, le Mercury recommanda d'amender la loi qui instituait deux cours d'appel. Toute loi sage n'en créait qu'une seule. Les juges du Bas-Canada devaient se réunir dans différentes parties de la province quatre fois par année. Comble d'absurdité, chaque juge en chef revoyait les jugements de l'autre. ${ }^{13}$ "As the Presidents live at the distance of near 200 miles from each other, they can have little opportunity to communicate together; and of course, uniformity and consistency in the administration of the law, cannot be expected." La rigueur du climat décuplait la difficulté pour les juges âgés: raison pour laquelle aucune session de la Cour d'appel n'avait eu lieu dans le district de Québec. ${ }^{14}$

d'obvier à ces maux possibles, et ici le chat sortait du sac, c'était de restreindre les juges à leur pouvoir judiciaire, de les exclure de l'Assemblée, de leur enlever tout pouvoir législatif, de mieux hiérarchiser les différentes cours, etc. (Le Canadien, 9 janvier 1808, etc.).

12 Le Canadien, 19 décembre 1807. Voir appendice V.

28.

13 Voir les observations de Sewell à ce propos dans notre Revue, XX:

14 The Quebec Mercury, 28 décembre 1807. 
D'ailleurs, le district de Québec n'était pas seul à souffrir de cette situation. Par suite de la mort du juge Davidson et d'un accident survenu au juge Monk, la Cour du Banc du roi de Montréal n'avait pu se rassembler pour sa session de mars 1807. Aussi Dunn délivra-t-il des commissions pour une cour d'Oyer et Terminer et pour l'évacuation générale des prisons. En conséquence, au cours de procès entre les 12 et 15 mai, John Smith et Joseph Barbary écopèrent d'une condamnation à mort, le premier pour avoir volé un bœuf, le second, un cheval. Or, contrairement à la loi, le juge en chef Monk, du district de Montréal, se contenta de remettre au président Dunn la liste des prisonniers jugés, sans y joindre des détails sur les procès et la procédure.

Consultés, le solliciteur général et le procureur général émirent des opinions diamétralement opposées. Le premier, Louis-Charles Foucher, argua que la loi de 1794 investissait la Cour du banc du roi d'une juridiction permanente dans les affaires criminelles. Hors de cette cour, une autre juridiction criminelle pouvait exister, mais délimitée par la commission d'Oyer et Terminer et pour l'évacuation générale des prisons. Dans chaque cas, tout jugement grave se trouvait automatiquement suspendu et sujet à ratification par l'administrateur civil de la colonie. La loi ne tolérait aucune exception. Bien plus, elle identifiait les catégories de personnes pouvant être emprisonnées par ce tribunal spécial. Les sentences étaient donc différées jusqu'à ce que Dunn se prononce: ce qu'il ne pouvait faire en connaissance de cause que si la cour le munissait de tous les renseignements pertinents. ${ }^{15}$

Le procureur général Sewell opina que tout s'était déroulé sous la seule commission d'Oyer et Terminer, sans référence à l'évacuation générale des prisons. Or, d'après la loi 34.G.III.c.6, une approbation spéciale des sentences par warrant sous le sceau de la province n'était requise que sous une commission d'Oyer et Terminer et pour l'évacuation générale des prisons. Par conséquent, Dunn n'avait pas besoin d'attendre le détail des procé-

15 Opinion du solliciteur général Foucher, 25 mai 1807, AC, M.G. 11, Q. 102: 322-325. 
dures des juges pour suspendre ou casser leur sentence et accorder un pardon aux deux condamnés à mort..16

Perplexe, Dunn se tourna vers le juge en chef Allcock. Ce dernier répondit brièvement que si le président graciait ces hommes, il n'avait pas auparavant à signifier son approbation de la sentence prononcée contre eux. ${ }^{17}$ Mais cette réponse ne satisfit pas Dunn. En effet, elle éludait la question de principe, savoir "whether the Provisions of the Act... with regard to Courts of Oyer and Terminer apply to the Commission under which Smith and Barbary were tried, and sentenced to suffer Death". Il s'en référait donc aux légistes anglais. En attendant, comme les crimes de Smith et Barbary ne nécessitaient pas une exécution immédiate, il les amnistierait en se fondant sur l'opinion du juge en chef. ${ }^{18}$

Les légistes britanniques endossèrent plutôt l'opinion de Foucher. Les clauses 4, 5 et 6 de la loi de 1794 s'appliquaient aux deux commissions - celle d'Oyer et Terminer, celle de l'évacuation générale des prisons -, émises le même jour aux mêmes personnes. Si l'on avait inséré les deux commissions dans le même acte, ces clauses "would, without any doubt, have applied to the Court sitting under them, and yet even then, the Court would in many cases of trial have derived its jurisdiction wholly from one of the Commissions, without any reference to the other, and we conceive that the circumstance of the two commissions being granted by separate instruments, can make no difference in the application of the Provincial Statute to the Court sitting under them." Toute sentence prononcée par une telle cour, affectant la vie ou les membres, demeurait inopérante jusqu'à ce que l'administrateur civil la sanctionne ou l'annule. Or il ne devrait pas, de façon générale, octroyer un pardon avant d'avoir scruté less détails des procédures de la cour dans le cas de la ou des personnes condamnées. Mais dans les circonstances

\footnotetext{
16 Opinion du procureur général Sewell, 19 mai 1807, ibid., 319-321.

17 Opinion du juge en chef Allcock, [s.d.], ibid., 329. ss.

18 Thomas Dunn à Windham (no 40), Québec, 10 juin 1807, ibid., 298
} 
particulières qui avaient confronté Dunn, "we think that it was prudent in him to grant such pardon, or at least to respite the Execution". ${ }^{19}$

On peut donc conclure, sans risquer de se tromper, que tout ne tournait pas rond dans l'administration de la justice. Des réformes structurelles s'imposaient afin d'accélérer les causes et les jugements, afin aussi d'enrayer le foisonnement de jurisprudences multiples et contradictoires. Il fallait en arriver à un système de jugements uniformes, basés sur les mêmes critères, c'est-à-dire les lois du pays et non pas l'équité ou les opinions personnelles et politiques des juges. Pareille métamorphose, surtout en ce qui avait trait au respect du droit français, ne s'opèrerait pas dans un avenir prochain. D'où le mécontentement à l'égard de l'administration de la justice, notamment chez les Canadiens, qui continuerait à suppurer longtemps comme un abcès chronique.

JeAN-PierRe WALLOT Musée national

\section{APPENDICE I}

Le Canadien, 11 juillet 1807.

Le lendemain 12 juin. - Mr. Perrault, Avocat, de la part de Madame veuve et héritiers Duchesnay et de l'exécuteur testamentaire, présenta à la Cour une requête exposant en substance, que comme le testament en question avait été prouvé devant la. Cour et qu'il contenait des substitutions, ils concluaient à ce qu'il plût à la. Cour ordonner que la publication du soi-dit testament fut faite immédiatement, audience tenante, et qu'ensuite il fût enregistré dans les registres de la Cour. - Mr. Perrault dit ensuite: J'ai une motion qui paraîtra peut-être extraordinaire, je demande que l'expédition du testament délivrée par le Greffier et celle délivrée par le Notaire soient publiées et enregistrées toutes deux; ma raison pour cela est que je suis informé qu'il y aura un appel du Jugement de cette Cour, et que comme il pourrait arriver qu'il serait renversé, mes parties se trouveraient toujours bien, parce que dans le cas de confirmation du Jugement, la publication de l'expédition du Notaire ne fera aucun mal; mais s'il était renversé, cela pourrait en faire. - Mr. Perrault, Greffier, lut le testament. Au nom du Père, \&c. - Le Greffier ayant lu le testament, jusqu'à la signature de Mr. Duchesnay inclusivement, allait lire la mention du dépôt fait chez le Notaire, qui est à la suite, laquelle commence par ce mot DEPOSE que le Greffier proféra, ensuite il arrêta. M. Panet demanda qu'il continuât, afin de conn̊ı̂tre quelle expédition il avait lue. - Mr.

19 Rapport des légistes de la couronne, V. Gibbs et Thos. Plumen, à Castlereagh, Lincoln's Inn, 28 août 1807, ibid., Q. 106-2: 515-517. 
Perrault, Greffier, dit: La Cour en dispense et dit au Juge en Chef: Les Ordonnances exigent que le testament soit lu et non pas l'acte de dépôt. - Mr. Panet dit: il faudrait que le Greffier dit qu'il a lu ce papier; mais se serait faux; car il n'a pas tout lu. - Mr. Williams dit qu'il était d'opinion que le dépôt de Me. Planté était irrégulier. - Mr. Panet dit: On doit constater laquelle des deux expéditions a été lue ou on doit les lire toutes deux. - Mr. Perrault, Greffier, dit que Mr. Panet n'avait pas droit de parler. - Mr. Panet dit qu'il avait droit comme un homme du public, qu'il fallait qu'on sût quelle expédition avait été lue, qu'il voulait savoir si c'était celle du Greffier ou du Notaire. - Le Juge en Chef dit: La Cour n'est pas pleine, il faut attendre à demain, (Mr. De Bonne manquait), alors Mr. Panet demandera que les deux expéditions soient lues. - Mr. Olivier Perrault dit: je demande que les deux soient lues pour plus de sûreté, en cas que l'une des deux expéditions fût déclarée illégale par la Cour d'Appel. Mr. Williams dit qu'iil pensait que ce serait contredire le jugement de la Cour. - Le Juge en chef dit que cela ne pouvait préjudicier, pourvu qu'on fit mention que ce serait EX MAJORE CAUTELA sans préjudice, \&c. Mr. Williams objectant, le Juge en Chef lui dit, s'il arrivait que l'expédition de Mr. Planté fût regardée comme seule valide, la publication serait encore bonne. Il ajouta qu'il fallait remettre au lendemain. - Mr. Perrault, Greffier, dit: Je suis d'opinion que c'est l'original qui doit être lu. - Mr. Panet dit que Pothier disait que c'était l'expédition. - Le Juge en Chef dit que tout ce qu'on pouvait demander c'était que le testament fût conservé que soit qu'il fût publié de manière ou d'autre, cela était indifférent, qu'il ne fallait pas s'arrêter à toutes ces petites formes.

Le lendemain 13 Juin. - Mr. Perrault, Greffier, lut ce qui suit, daté sur le registre de la veille, c'est-à-dire du 12. Consorts.

Sur requête de Dme C. Dupré, veuve de l'Hon. Ant. Juch. Duchesnay \&

La Cour ayant égard à ladite requête tendante à ce que le testament de feu l'Honorable Antoine Juchereau Duchesnay du 24 décembre 1802, reconnu et prouvé devant cette Cour le trois de ce mois, soit publié et enregistré, ordonne que l'expédition d'icelui délivrée par le Greffier de cette Cour, soit maintenant lue et registrée ès régistres des insinuations des substitutions au Greffe de cette Cour. En conséquence l'expédition délivrée par le Greffe de cette Cour du testament de feu l'Hon. Ant. Juch. Duchesnay du 24 déc. 1802 a été publiée en jugement ce jourd'hui, Cour tenante, et enregistré ès régistres régistres [ sic] des insinuations des substitutions du Greffe de cette Cour.

La Cour mit en délibéré sur la motion pour faire publier et enrégistrer une expédition du testament de l'Hon. Duchesnay délivrée par $\mathbf{M r}$. Jh. Planté. - Fr. Perrault, Greffier, lut en outre ce qui suit daté sur le registre du 13: "La Cour ayant mûrement délibérée sur la motion du suppliant tendante à faire publier et enregistrer une expédition du testament de feu l'Hon. Ant. Juch. Duchesnay délivrée par Mr. Jh. Planté, rejette ladite motion."

Un registre neuf et paraphé le même jour par un des Juges de la Cour par premier et dernier feuillet a été étrenné par l'enregistrement dudit testament dans les termes suivants: "En conséquence de l'ordre de la Cour du Banc du Roi pour le district de Québec du 12 Juin 1807, lue la requête de Dame Catherine Dupré, veuve de l'Honorable Antoine Juchereau Duchesnay, et consorts, le testament dudit feu Hon. Duchesnay du 24 Décembre 1802, expédié par Jh. Fr. Perrault, Greffier et Prothonotaire de cette dite Cour, lu en jugement ledit jour, Cour tenante, a été enregistré 
dans le présent registre pour servir à l'insinuation des substitutions de ce district comme suit:"

Après est la teneure du testament, sans aucune mention du dépôt, et ensuite celle des dépositions de Messieurs Planté, Têtu \& Berthelot, dont mention est ci-devant faite.

\section{A.PPENDICE II}

FIN.

Le Canadien, 1er août 1807.

... Cette décision [de la Cour d'appel] est importante, $1^{\circ}$. parce qu'elle semble établir que les anciennes formes de procéder, qui n'ont pas été abrogées par l'ordonnance de 1785 , sont en force, contre l'opinion de $\mathrm{Mr}$. le Juge en Chef Elmsley, qui a dit plusieurs fois que la conquête avait anéanti toutes les formes de procéder des anciennes Cours comme elle avait anéanti ces Cours elles-mêmes, et que le fonds des anciennes lois avait seul été conservé et rétabli par l'acte de Québec de 1774. Cette décision de la Cour d'appel paraît avoir établi formellement le contraire de cette opinion; car la fixation de l'époque qui termine le temps dans lequel on doit faire une opposition, fait certainement partie des lois de forme, de même que la fixation du temps dans lequel on doit faire toute autre procédure. Ce n'est pas parce que cette époque était fixée par la couture de Paris même qu'on doit la regarder comme faisant partie du fonds du droit, parce que c'est par la nature de la loi même et non pas par le titre du livre où elle est écrite qu'on doit juger si elle fait partie de la forme ou du fonds du droit.

$2^{\circ}$. Cette décision semble établir que les Cours de Justice ne peuvent faire de Règles de Pratique qui soient contraires non seulement à la lettre, mais à l'esprit même et aux principes de ces anciennes lois de forme; car dans le cas présent il ne s'agissait que de l'esprit de l'ancienne loi... La Cour d'Appel a considéré que la Règle de la Cour de Montréal était nulle, parce que dans la fixation qu'elle avait faite de l'époque, elle s'était écartée du principe de l'ancienne loi... D'où il semble qu'on doit tenir pour principe certain d'après cette décision, $1^{\circ}$. Que les anciennes lois qui règlent les formes de procéder qui n'ont pas été expressément abrogées sont en force. $2^{\circ}$. Que toutes les Règles des Cours de Justice de cette Province qui ne sont pas faites d'une manière aussi analogue que possible aux principes de celles de ces anciennes lois dont la lettre n'est pas en force, sont nulles de plein droit.

\section{APPENDICE III}

\section{Monsieur le Rédacteur du Canadien.}

Le Canadien, 21 novembre 1807.

Les questions que vous avez bien voulu suggérer dans votre dernier numéro, me semble, doivent attirer l'attention de toutes personnes éclairées, et surtout de celles qui ont le bonheur de leur Patrie à cour; quant à moi, quoique mes talents soient bien au-dessous de cette tâche, je vais entreprendre de répondre à la sixième question, ce n'est que pour le bonheur de mes compatriotes que j'ose hasarder une pareille entreprise que de paraître sur un papier public, pour répondre à une question aussi importante que celle qui demande le meilleur plan pour l'administration de la Justice dans la Province. Il sera inutile pour moi d'entrer dans les détails de l'importance de cette administration, il me suffira de dire que la due administration de la Justice dans un Etat est le plus grand support de son gouverne- 
ment; car sous notre heureuse Constitution (celle de la Grande Bretagne) c'est le Souverain lui-même qui administre ses lois à son peuple par le moyen de ses Cours de Justice. Nos lois, pour parler généralement, sont les meilleures du monde, mais quant à la manière de les administrer il n'y a point de pays dans l'univers où la Justice est peut-être plus mal administrée, par manque d'organisation convenable. Tantôt on vous juge les affaires par équité, tantôt par les lois Françaises, tantôt par les lois Anglaises. Pour preuve des défaults il me sera seulement nécessaire de vous dire que dans deux causes, dont l'une était au-dessus de vingt-livres sterlings et l'autre au-dessous ${ }^{1}$ et, où la question qui s'est présentée était la même, il y a eu deux jugements rendus, tout-à-fait différents. Il se glisse aussi nombre d'abus dans nos Cours. Je puis vous assurer encore que tous nos Juges diffèrent d'opinion sur la manière d'admettre la preuve testimoniale pour les sommes excédant cent livres tournois. Mais je vous en ai dit assez du mal qui règne, il faut $\mathrm{y}$ porter remède et répondre à la question. Quel est le meilleur plan pour l'administration de la justice dans la Province? Je parlerai seulement des districts de Québec et Montréal. La Cour d'Appel serait composée de cinq Juges (outre les Conseillers qui y conserveraient leurs sièges) dont le Juge en Chef de la Province serait un et les quatre autres seraient les meilleurs Jurisconsultes du pays, cette Cour siégerait six fois par année et entendrait et reviserait tous les Jugements des Cours du Banc du Roi dans leurs termes supérieurs, dans les différents districts sans exception, et je voudrais aussi régler les frais des Appels, afin de les faciliter à des pauvres malheureux qui auraient pu être ruinés par les Jugements de la Cour Inférieure, et qui faute des moyens nécessaires deviendraient la proie de leurs semblables. La Cour du Banc du Roi pour les Causes Civiles serait composée de trois Juges (parce que, lorsqu'il y en a quatre, la Cour peut se trouver divisée et par là empêcher le cours de la Justice) dont le Juge en Chef du district serait un, et pour les causes criminelles je ferais présider cette Cour par le Juge en Chef de la Province et en son absence par le Juge en Chef du district. Quant à la Cour du Banc du Roi Civile, je voudrais diriger ses pouvoirs par un acte du Parlement, dont elle ne pût s'écarter et je voudrais lui ôter tous ses pouvoirs législatifs et par là empêcher nombre d'abus qui se glissent dans cette partie de l'administration, entr'autres celui qu'ils prennent sur eux, en faisant des règles de Pratique qui abrègent des lois positives; voyons ces Règles avec l'Ordonnance de 1687 , et nous verrons si par là on a point abrogé une grande partie de cette Ordonnance. Rien n'est plus dangereux, suivant moi, que de donner un pouvoir législatif à des Cours de Justice. Je pourrais citer nombre d'autres exemples; mais je crois que ce que j'en ai dit sur ce point est suffisant. Quant aux Jurisdictions Inférieures, je me propose de vous envoyer mes idées sur le plan de leur administration en temps pour quelqu'autre de vos Numéros.

Montréal, 13 novembre, 1807.

Un Amateur de la Justice.

Le Canadien, 12 décembre 1807.

(Continuation de l'Amateur de la Justice.)

Les Cours inférieures pour les causes de dix livres sterlings et audessous seraient tenues par un seul Juge qui jugerait seulement dans ces

1 Il n'y a point d'appel des Causes au-dessous de vingt-livres sterlings, et la Cour du Banc du Roi en juge finalement. 
Cours, les termes seraient les rnêmes qu'ils sont actuellement, et quant à la manière de juger les causes, elles seraient jugées suivant la loi du pays et non suivant l'équité, car sous ce prétexte les Cours peuvent donner des jugements qui sont contraires à la loi et au bon sens; il ne me restera done qu'à parler de la manière dont je voudrais établir les Cours de Quartiers de Sessions et de semaine. - Quant à la première je voudrais nommer trois Juges de Paix, qui eussent des connaissances suffisantes de la loi, et leur donner un appointement convenable pour qu'il ne s'occupassent d'autres affaires que celles des Quartiers de sessions. Je voudrais enfin ôter à cette Cour les pouvoirs législatifs qu'elle a, car elle peut établir des lois sous le titre de Règlements de Police qui soient contraires aux voix du peuple, et elle l'a fait. Je citerai dans ce cas un exemple des plus frappants: c'est que la Chambre d'Assemblée de cette Province, après s'être bien occupée d'un Bill pour empêcher les Inhumations dans les Villes, et après tous les térnoignages et informations qu'elle a pris, elle a rejetté ce bill, et les Magistrats de cette ville aussitôt la rejection d'icelui, qu'ont-ils fait ? Oserais-je dire, que contre la voix du peuple, par leurs représentants dont quelques-uns étaient de ces mêmes représentants, ils ont fait un Règlement de Police qui avait le même effet que ce Bill, qui avait été rejetté de la Chambre avec tant de raison, d'où on peut conclure que lorsqu'il y a dans la Chambre seulement un Juge de Paix et qu'il aurait le dessous dans un bill qui pourrait concerner la Police, il (car il peut avoir tant d'influence sur ses confrères Magistrats) peut faire passer des Règlements qui seraient contraires à la volonté du peuple, et cela pour que cet individu puisse dire que ses pouvoirs excèdent même ceux du Parlement, qui a les pouvoirs les plus arbitraires, et en les privant de ces pouvoirs ce serait remédier au mal qui existe. Quant aux Sessions de Semaine, je voudrais nommer deux Juges de Paix pour cette Cour, aussi avec un appointement, et elle serait présidée par un des trois Juges de la Cour des Quartiers de Sessions, car il résulte de grands abus en n'ayant point de Cour de Semaine fixe ou de Juges qui soient obligés de la tenir; ces Messieurs étant ordinairement des Notaires ou personnes d'autres professions, ils ne sont pas toujours en état de pouvoir laisser leurs propres affaires pour celles du public, quelque soit leur bonne volonté, et de là il provient que les trois quarts du temps il n'y a point de Cour, et les personnes qui y ont fait des poursuites perdent leur temps et déboursent, et la justice n'est pas administrée. - Voilà de quelle manière je voudrais établir des Cours de Justices dans le district de Québec et de Montréal, et par là j'espérerais que la Justice serait mieux administrée, et s'il y avait malheureusement, ce que je ne puis croire, des Juges dans ce pays tels que celui dont parle l'OBSERVAT'EUR, (inséré dans le $50 \mathrm{e}$ No. du 1 er vol.) que le Parlement de la Province se serve donc du droit qu'il a de faire une adresse à la personne ayant l'administration du gouvernement pour les faire déplacer, et le Gouverneur ou la personne ayant cette administration est obligé de le faire, lorsque cette adresse est faite par les deux tiers de la Chambre.

30 Novembre, 1807.

UN AMATEUR DE LA JUSTICE.

\section{APPENDICE IV}

Le Canadien, 26 décembre 1807.

... Dites-moi ... d'où vous puisez cet avancé de votre écrit [obligation du gouverneur de déplacer us juge, à la demande des deux tiers de la Chambre d'Assemblée]... Pour moi je ne connais rien dans la Constitu- 
tion qui justifie cette proposition... Je sais bien qu'en Angleterre, afin de conserver la dignité et l'indépendance des Juges des Cours Supérieures, il est statué par un acte du Parlement que leurs commissions ne seront pas, comme autrefois, durant plaisir, mais durant bonne conduite, que le Roi peut cependant les déplacer sur l'adresse des deux Chambres du Parlement. En ce pays, où les commissions des Juges ne sont que durant plaisir, le Roi ou son représentant peut les déplacer sans adresse,... [ou] sur l'adresse des deux Chambres du Parlement Provincial, ou même sur l'adresse de la Chambre d'Assemblée seule, cela s'entend non seulement des deux tiers, mais même de la majorité de la Chambre. Je n'avancerai pas... que le Roi ou le Gouverneur... soit obligé de le faire, mais... [l'un ou l'autre] trouvera convenable de déplacer un Juge sur l'adresse que lui aura présentée la Chambre d'Assemblée, après une investigation légale de la mauvaise conduite, du mauvais caractère, de la mauvaise réputation, de la partialité ou de l'ignorance de tel Juge.

UN SOU'SCRIPTEUR.

\section{APPENDICE V}

Le Canadien, 19 décembre 1807.

Monsieur le Rédacteur.

J'ai souvent réfléchi sur les inconvénients qui résultent de l'organisation de nos cours de Justice, et surtout de leur établissement par termes éloignés les uns des autres.

On se plaint de l'esprit de chicane des habitants de notre Province, ces plaintes sont assez mal fondées. On se récrie surtout sur la multitude des causes dans les Termes Inférieurs dans le district de Montréal. Mais il est impossible qu'elles ne s'y accumulent pas à l'excès, il n'y a qu'une cour pour toutes les affaires civiles au-dessous et dix Livres Sterling où tous les habitants du district à quelque distance qu'ils soient de la Ville où le siège de la Justice est établi, sont obligés de venir discuter leurs droits, et il faut que cette Cour seule subvienne aux besoins d'une population de cent mille habitants dispersés sur une étendue de plusieurs centaines de milles. Encore ne se tient-elle que cinq fois dans l'année, huit jours de suite, une autre six, et est-elle interrompue par les fêtes et les dimanches qui en retranchent souvent deux ou trois sur le nombre. Pour la façon d'un fossé ou la valeur d'une clôture de quelques shellings, si un habitant a besoin de forcer un voisin déraisonnable à des travaux nécessaires et communs, sans parler des autres, à la fin de Mai, il faut attendre au mois de Juin; et s'il n'a pas le bonheur d'être un des premiers sur les rangs, il est clair qu'il lui faut aller au mois de Septembre, temps où les causes du circuit, qui, je dois dire en passant, presque inutile de l'aveu de tous ceux qui ont quelqu'expérience, encombrent la Cour et rendent plus difficile d'obtenir une décision dans ce terme. C'est une espèce de miracle s'il peut obtenir Jugement à cette époque lorsque toutes les espérances de récolte pour cette année sont déjà perdues. Encore s'il a un adversaire un peu adroit est-il très douteux qu'il ne soit pas obligé de revenir en Novembre, temps où les travaux qu'il a demandés sont devenus inutiles et ne peuvent plus se faire. Heureux encore quand il n'a pas dépensé le revenu de sa terre en frais, voyages, pensions, témoins, experts, \&c, \&c. Il en est à peu près de même pour les personnes de la Ville qui vivent du revenu de leurs fonds. Un propriétaire de maison veut-il chasser un locataire mauvais payeur au premier de Mai, il est obligé de sommer pour le 21 Mai au terme Inférieur et au 1er de Juin dans les Cours supérieures. Si le débiteur est de mauvaise foi et nie la dette il faut faire preuve, le 
terme se passe, on est rendu au terme suivant et l'on rit des efforts du propriétaire pour soutenir ses droits contre un usurpateur. Avez-vous obtenu Jugement? Nouvelle difficulté. Il faut en saisissant appointer un gardien à grand frais. Une tierce personne interposée met opposition à la saisie, il faut attendre au terme suivant où vous obtenez Jugement si vous pouvez, sinon il faut encore attendre quelques mois après et il vous reste alors ce que les frais d'exécution, de saisie et de garde n'ont pas absorbé. Heureux quand vous n'êtes pas obligé de payer de vos deniers l'Officier que vous avez employé pour faire la besogne. Il en est de même de toutes les autres créances.

Le recouvrement des dettes de commerce les plus communes et les moins litigieuses, est accompagné des mêmes difficultés, quand le débiteur veut employer ses moyens. Il est presque vrai de dire que souvent on obtient jugement contre ses débiteurs parce qu'ils veulent bien y consentir. Il faut aussi remarquer que la multitude des causes qui s'accumulent par ce moyen, met le Juge, quelque sagacité qu'on lui suppose, hors d'état de donner à ses décisions toute l'attention nécessaire, les Avocats de rendre justice à leurs clients dans la conduite de leur cause, et fournit en outre des moyens faciles à tous les plaideurs de mauvaise foi ou Chicaneurs d'éluder la Justice. L'espérance de l'impunité les autorise, le peu qu'il en coûte pour y parvenir est un motif de plus pour spéculer en ce genre.

Je voudrais finir par dire qu'une Cour Hebdomadaire remédierait à tous ces inconvénients ou au moins au plus grand nombre et aux plus essentiels, mais c'est une vieille idée rebattue. J'ai peut-être déjà scandalisé bien des oreilles délicates et chatouilleuses. J'y reviendrai pourtant un de ces jours.

UN AMI DU BIEN. 